\title{
Projection algorithms with dynamic stepsize for constrained composite minimization
}

\author{
Yujing Wua ${ }^{\mathrm{a}}$, Luoyi Shi ${ }^{\mathrm{b}, *}$, Rudong Chen ${ }^{\mathrm{b}}$ \\ ${ }^{a}$ Tianjin Vocational Institute, Tianjin 300410, P. R. China. \\ ${ }^{b}$ Department of Mathematics, Tianjin Polytechnic University, Tianjin 300387, P. R. China.
}

Communicated by Y. H. Yao

\begin{abstract}
The problem of minimizing the sum of a large number of component functions over the intersection of a finite family of closed convex subsets of a Hilbert space is researched in the present paper. In the case of the number of the component functions is huge, the incremental projection methods are frequently used. Recently, we have proposed a new incremental gradient projection algorithm for this optimization problem. The new algorithm is parameterized by a single nonnegative constant $\mu$. And the algorithm is proved to converge to an optimal solution if the dimensional of the Hilbert space is finite the step size is diminishing (such as $\alpha_{n}=\mathcal{O}(1 / n)$ ). In this paper, the algorithm is modified by employing the constant and the dynamic stepsize, and the corresponding convergence properties are analyzed.
\end{abstract}

Keywords: Composite minimization, projection algorithm, dynamic stepsize.

2010 MSC: 47H10, 54H25.

(C)2018 All rights reserved.

\section{Introduction}

We consider a constrained optimization problem where the objective is a sum of component functions:

$$
\min _{x \in C:=\cap_{i=1}^{N} C_{i}} f(x)=\sum_{j=1}^{M} f_{j}(x),
$$

where $N$ and $M$ are large integers, $\left\{C_{i}\right\}$ are finite nonempty closed convex subsets of a Hilbert space $H$, and $f_{j}: H \rightarrow \mathbb{R}$ are convex and differentiable functions. Problem of the form (1.1) arises in lots of applied areas, and it is of central importance in machine learning and statics, for more details see $[3,10,20]$.

\footnotetext{
*Corresponding author

Email addresses: xiaomi20062008@163.com (Yujing Wu), shiluoyi@tjpu.edu.cn (Luoyi Shi), chenrd@tjpu.edu.cn (Rudong Chen)
}

doi: $10.22436 /$ jnsa.011.07.05

Received: 2017-12-25 Revised: 2018-04-13 Accepted: 2018-04-14 
For the unconstrained composite minimization problem (i.e., $\mathrm{C}_{\mathrm{i}}=\mathrm{H}$ ), gradient-like incremental methods are also frequently used, when and the number of the component functions is large. The incremental gradient algorithm (IGA) [3] is similar to the classical subgradient algorithm: if $x_{n}$ is constructed, let

$$
\psi_{0}=x_{n}, \psi_{j}=\psi_{j-1}-\alpha_{n} \nabla f_{j}\left(\psi_{j-1}\right), j=1,2, \ldots, M, \quad x_{n+1}=\psi_{M},
$$

where $\alpha_{n}$ is a positive step size. It is easy to check that the IGA has the form

$$
x_{n+1}=x_{n}-\alpha_{n} \sum_{j=1}^{M} \nabla f_{j}\left(\psi_{j-1}\right) .
$$

Note that, when the component functions $f_{j}$ and their gradients are evaluated at the same vector $x_{n}$, then the above algorithm is the classical steepest descent algorithm (SDA) [3]:

$$
x_{n+1}=x_{n}-\alpha_{n} \sum_{j=1}^{M} \nabla f_{j}\left(x_{n}\right) .
$$

More general versions of incremental subgradient methods can be seen in [4, 11, 12, 15, 23].

In [22], Yang and $\mathrm{Xu}$ proposed the following projection algorithm for the problem (1.1) where the step size is diminishing (i.e., $\left.\alpha_{n}=\mathcal{O}(1 / n)\right)$ : Choose an initial value $x_{0} \in H$ arbitrarily, then iterate $x_{n+1}(n>0)$ is as follows:

$$
\left\{\begin{array}{l}
x_{n, 0}=x_{n} \\
x_{n, j}=x_{n, j}-\alpha_{n} \nabla f_{n}\left(x_{n, j-1}\right), j=1,2, \ldots, M \\
x_{n+1}=\sum_{i=1}^{N} \beta_{i} P_{C_{i}}\left(x_{n, M}\right),
\end{array}\right.
$$

where $P_{C_{i}}$ is the projection from $H$ to $C_{i}$ for each $1 \leqslant i \leqslant N$, and $\beta_{i}>0$ is such that $\sum_{i=1}^{N} \beta_{i}=1$. And get that: (1) if the dimensional of the Hilbert space is finite then $\left\{x_{n}\right\}$ converges to an optimal solution of the problem (1.1); (2) if the dimensional of the Hilbert space is infinite and the limit of the sequence $\left\{f\left(x_{n}\right)\right\}$ exists, then $\left\{x_{n}\right\}$ converges weakly to an optimal solution of the problem (1.1)

Recently, we [19] modified the algorithm (1.2) by a single nonnegative constant $\mu$ as follows:

Algorithm 1.1. Let $\mu$ is a fixed scalar $\mu>0$, choose an initial value $x_{0} \in H$ arbitrarily, then iterate $x_{n+1}(n>0)$ is as follows:

$$
\left\{\begin{array}{l}
x_{n, 0}=x_{n} \\
x_{n, j}=x_{n}-\alpha_{n} h_{j}, j=1,2, \ldots, M \\
h_{j}=\sum_{k=1}^{j} \omega_{k, j}(\mu) \nabla f_{k}\left(x_{n, k-1}\right), j=1,2, \ldots, M \\
x_{n+1}=\sum_{i=1}^{N} \beta_{i} P_{C_{i}}\left(x_{n, M}\right),
\end{array}\right.
$$

where

$$
\omega_{k, j}(\mu)=\frac{1+\mu+\cdots+\mu^{j-k}}{1+\mu+\cdots+\mu^{M-k}}, j=1,2, \ldots, \quad M, 1 \leqslant k \leqslant j,
$$

step size $\alpha_{n}>0, P_{C_{i}}$ is the projection from $H$ to $C_{i}$ for each $1 \leqslant i \leqslant N$, and $\beta_{i}>0$ is such that $\sum_{i=1}^{N} \beta_{i}=1$. In [19], we get the same convergence properties with [22].

Note that, in the algorithm (1.3) $\omega_{k, M}=1, k=1,2, \ldots, M$, it follows that

$$
x_{n, M}=x_{n}-\alpha_{n} h_{M}=x_{n}-\alpha_{n} \sum_{j=1}^{M} \nabla f_{j}\left(x_{n, j-1}\right) .
$$

If $\mu=0$, then $\omega_{k, j}=1$ for all $k$ and $j$. If $\mu \rightarrow \infty$, we have $\omega_{k, j} \rightarrow 0$ for all $i$ and $j$, and $x_{n, j} \rightarrow x_{n}$ 
for $j=1,2, \ldots, M$. Hence, the the algorithm (1.3) reduces to (1.2) when $\mu=0$. Furthermore, for the constrained composite minimization problem with $N=1$, and the extreme values $\mu=0, \mu=\infty$, the algorithm is obtained as special cases the algorithm IGA in [14] and algorithm SDA in [15] , respectively; for the unconstrained composite minimization problem (i.e., $C_{i}=H, i=1,2, \ldots, N$ ), and the extreme values $\mu=0, \mu=\infty$, the algorithm is obtained as special cases of algorithms IGA and SDA in [3], respectively. For $N=M=1$, the algorithm is obtained as the special case of algorithm GPA in [21].

The purpose of this paper is modifying the Algorithm 1.1 by employing the constant and the dynamic stepsizes. And we research the convergence properties of Algorithm 1.1 for there types of stepsize rules: constant stepsize, dynamic stepsize for known the optimal value $f^{*}$, and dynamic stepsize for unknown $f^{*}$. For case of dynamic stepsize for known $f^{*}$, we get that the Algorithms 1.1 converges weakly to an optimal solution $x^{*}$ of the problem (1.1).

\section{Preliminaries}

In this section we shall give a few preliminary definitions and lemmas which are important in the prove of our main theorem .

Let $\mathrm{P}_{\mathrm{C}}$ denote the projection from $\mathrm{H}$ onto a nonempty closed convex subset $\mathrm{C}$ of $\mathrm{H}$; that is,

$$
P_{C}(x)=\min _{y \in C}\|x-y\| .
$$

It is well known that $\mathrm{P}_{\mathrm{C}}(\mathrm{x})$ is nonexpansive and is characterized by the inequality

$$
\left\langle x-P_{C}(x), y-P_{C}(x)\right\rangle \leqslant 0, \forall y \in C .
$$

Moreover,

$$
\left\|P_{C}(x-y)\right\|^{2} \leqslant\|x-y\|^{2}-\left\|P_{C} x-x\right\|^{2}, \forall x \in H, y \in C .
$$

Let $S=\left\{x^{*} \in C=\cap_{i=1}^{N} C_{i}, f\left(x^{*}\right)=\inf _{x \in C} f(x)\right\}$ be the set of optimal solution of the problem (1.1) and $f^{*}=\inf _{x \in C} f(x)$ be the optimal value. We always assume consistency of the problem of (1.1) from now on, that is to say $S \neq \emptyset$.

We now collect some elementary facts which will be used in the proofs of our main results.

Lemma 2.1 ([7, 8]). Let X be a Banach space, $\mathrm{C}$ a closed convex subset of $\mathrm{X}$, and $\mathrm{T}: \mathrm{C} \rightarrow \mathrm{C}$ a nonexpansive mapping with $\operatorname{Fix}(\mathrm{T}) \neq \emptyset$. If $\left\{\mathrm{x}_{\mathrm{n}}\right\}$ is a sequence in $\mathrm{C}$ weakly converging to $\mathrm{x}$ and if $\left\{(\mathrm{I}-\mathrm{T}) \mathrm{x}_{\mathrm{n}}\right\}$ converges strongly to $\mathrm{y}$, then $(\mathrm{I}-\mathrm{T}) \mathrm{x}=\mathrm{y}$.

Lemma 2.2 ([18]). Let $\mathrm{H}$ be a Hilbert space and $\left\{x_{\mathrm{n}}\right\}$ a sequence in $\mathrm{H}$ such that there exists a nonempty set $\mathrm{K} \subseteq \mathrm{H}$ satisfying the following

(i) for every $x \in K, \lim _{n \rightarrow \infty}\left\|x_{n}-x\right\|$ exists;

(ii) any weak-cluster point of the sequence $\left\{x_{n}\right\}$ belongs to $C$.

Then, there exists $\tilde{x} \in \mathrm{K}$ such that $\left\{x_{\mathrm{n}}\right\}$ weakly converges to $\tilde{x}$.

The following Lemma [19, Prop. 3.3] will be used repeatedly in the subsequent convergence analysis. And we include it for the sake of completeness.

Lemma 2.3. Let $\left\{x_{n}\right\}$ be generated by the Algorithm 1.1. Assume that there exists a positive constant $\mathrm{L}>0$ such that

$$
\left\|\nabla f_{j}\left(x_{n, j-1}\right)\right\| \leqslant L, j=1,2, \ldots, M, n \geqslant 1
$$

Then,

$$
\left\|x_{n+1}-x\right\|^{2} \leqslant\left\|x_{n}-x\right\|^{2}-2 \alpha_{n}\left[f\left(x_{n}\right)-f(x)\right]+5 \alpha_{n}^{2} M^{2} L^{2}
$$

for any $x \in \mathrm{C}=\cap_{i=1}^{\mathrm{N}} \mathrm{C}_{i}$. 
Proof. By the algorithm, for $1 \leqslant j \leqslant M$,

$$
\left\|x_{n, j}-x_{n}\right\|=\alpha_{n}\left\|h_{j}\right\|=\alpha_{n}\left\|\sum_{k=1}^{j} \omega_{k, j}(\mu) \nabla f_{k}\left(x_{n, k-1}\right)\right\| \leqslant \alpha_{n} M L .
$$

Since the projections $\mathrm{P}_{\mathrm{C}_{i}}$ are nonexpansive, and using the convexity of the norm, we have, for any $x \in C$,

$$
\begin{aligned}
\left\|x_{n+1}-x\right\|^{2} \leqslant & \sum_{i=1}^{N} \beta_{i}\left\|P_{C_{i}} x_{n, M}-P_{C_{i}} x\right\|^{2} \\
\leqslant & \left\|x_{n, M}-x\right\|^{2} \\
= & \left\|x_{n}-x-\alpha_{n} \sum_{j=1}^{M} \nabla f_{j}\left(x_{n, j-1}\right)\right\|^{2} \\
\leqslant & \left\|x_{n}-x\right\|^{2}-2 \alpha_{n}\left\langle\sum_{j=1}^{M} \nabla f_{j}\left(x_{n, j-1}\right), x_{n}-x\right\rangle+\alpha_{n}^{2} M^{2} L^{2} \\
= & \left\|x_{n}-x\right\|^{2}-2 \alpha_{n}\left\langle\sum_{j=1}^{M} \nabla f_{j}\left(x_{n, j-1}\right), x_{n, j-1}-x\right\rangle+\alpha_{n}^{2} M^{2} L^{2} \\
& +2 \alpha_{n}\left\langle\sum_{j=1}^{M} \nabla f_{j}\left(x_{n, j-1}\right), x_{n, j-1}-x_{n}\right\rangle \\
\leqslant & \left\|x_{n}-x\right\|^{2}-2 \alpha_{n}\left\langle\sum_{j=1}^{M} \nabla f_{j}\left(x_{n, j-1}\right), x_{n, j-1}-x\right\rangle+3 \alpha_{n}^{2} M^{2} L^{2} .
\end{aligned}
$$

Note that

$$
f_{j}(x) \geqslant f_{j}\left(x_{n, j-1}\right)+\left\langle\nabla f_{j}\left(x_{n, j-1}\right), x-x_{n, j-1}\right\rangle .
$$

We get that

$$
\begin{aligned}
\left\|x_{n+1}-x\right\|^{2} & \leqslant\left\|x_{n}-x\right\|^{2}-2 \alpha_{n} \sum_{j=1}^{M}\left(f_{j}\left(x_{n, j-1}\right)-f_{j}(x)\right)+3 \alpha_{n}^{2} M^{2} L^{2} \\
& =\left\|x_{n}-x\right\|^{2}-2 \alpha_{n} \sum_{j=1}^{M}\left(f_{j}\left(x_{n}\right)-f_{j}(x)\right)+3 \alpha_{n}^{2} M^{2} L^{2}-2 \alpha_{n}\left(\sum_{j=1}^{M} f_{j}\left(x_{n, j}\right)-f_{j}\left(x_{n}\right)\right) .
\end{aligned}
$$

Using

it follows that

$$
f_{j}\left(x_{n, j}\right)-f_{j}\left(x_{n}\right) \geqslant\left\langle\nabla f_{j}\left(x_{n}\right), x_{n, j}-x_{n}\right\rangle \geqslant-L\left\|x_{n, j}-x_{n}\right\|,
$$

$$
\begin{aligned}
\left\|x_{n+1}-x\right\|^{2} & \leqslant\left\|x_{n}-x\right\|^{2}-2 \alpha_{n} \sum_{j=1}^{M}\left(f_{j}\left(x_{n}\right)-f_{j}(x)\right)+3 \alpha_{n}^{2} M^{2} L^{2}+2 \alpha_{n} L\left(\sum_{j=1}^{M}\left\|x_{n, j}-x_{n}\right\|\right) \\
& \leqslant\left\|x_{n}-x\right\|^{2}-2 \alpha_{n} \sum_{j=1}^{M}\left(f_{j}\left(x_{n}\right)-f_{j}(x)\right)+5 \alpha_{n}^{2} M^{2} L^{2} \\
& =\left\|x_{n}-x\right\|^{2}-2 \alpha_{n}\left(f\left(x_{n}\right)-f(x)\right)+5 \alpha_{n}^{2} M^{2} L^{2} .
\end{aligned}
$$

That is to say

$$
\left\|x_{n+1}-x\right\|^{2} \leqslant\left\|x_{n}-x\right\|^{2}-2 \alpha_{n}\left(f\left(x_{n}\right)-f(x)\right)+5 \alpha_{n}^{2} M^{2} L^{2} .
$$




\section{Convergence analysis for there types of stepsize}

In this section, we research the convergence properties of Algorithm 1.1 for there types of stepsize rules: constant stepsize, dynamic stepsize for known $f^{*}$, and dynamic stepsize for unknown $f^{*}$.

\subsection{Constant stepsize}

We first research the case of constant stepsize, that is to say the stepsize $\alpha_{n}$ in the Algorithm 1.1 is fixed to a positive constant.

Proposition 3.1. Let $\left\{x_{n}\right\}$ be generated by the Algorithm 1.1 with the stepsize $\left\{\alpha_{n}\right\}$ fixed to a positive constant $\alpha$. And assume the boundedness of gradient (i.e., (2.1) is met). Then we have:

(i) if $\mathrm{f}^{*}>-\infty$, then $\liminf _{n \rightarrow \infty} f\left(x_{n}\right) \leqslant f^{*}+\frac{5 \alpha M^{2} L^{2}}{2}$, where $L$ is given in (2.1) and $M$ is the number of component functions;

(ii) if $f^{*}=-\infty$, then $\liminf _{n \rightarrow \infty} f\left(x_{n}\right)=-\infty$.

Proof. Assume (i) is not hold, then there exits $\varepsilon_{0}>$ such that

$$
\liminf _{n \rightarrow \infty} f\left(x_{n}\right)>f^{*}+\frac{5 \alpha M^{2} L^{2}}{2}+2 \varepsilon_{0} .
$$

It follows that there exists $\tilde{x} \in \mathrm{C}=\cap_{i=1}^{N} C_{i}$ such that

$$
\liminf _{n \rightarrow \infty} f\left(x_{n}\right)>f(\tilde{x})+\frac{5 \alpha M^{2} L^{2}}{2}+2 \varepsilon_{0}
$$

Hence, there exists $\mathrm{N}>0$ such that

$$
f\left(x_{n}\right)>f(\tilde{x})+\frac{5 \alpha M^{2} L^{2}}{2}+\varepsilon_{0}
$$

when $n>N$. Choose $x=\tilde{x}$ in Lemma 2.3 with the above inequality, we can get that

$$
\begin{aligned}
\left\|x_{n+1}-\tilde{x}\right\|^{2} & \leqslant\left\|x_{n}-\tilde{x}\right\|^{2}-2 \alpha\left(f\left(x_{n}\right)-f(\tilde{x})\right)+5 \alpha^{2} M^{2} L^{2} \\
& \leqslant\left\|x_{n}-\tilde{x}\right\|^{2}-2 \alpha \varepsilon_{0} \\
& \leqslant\left\|x_{n-1}-\tilde{x}\right\|^{2}-4 \alpha \varepsilon_{0} \\
& \vdots \\
& \leqslant\left\|x_{N}-\tilde{x}\right\|^{2}-2(n+1-N) \alpha \varepsilon_{0}
\end{aligned}
$$

when $n>N$, which is impossible for sufficiently large $n$ and hence it's a contradiction. So conclusion (i) is hold.

By a minor modification of above proof, conclusion (ii) is hold.

\subsection{Dynamic stepsize for known $\mathrm{f}^{*}$}

In this subsection, we analyze the convergence properties of Algorithms 1.1 for dynamic stepsize with $f^{*}$ being known. The dynamic stepsize $\left\{\alpha_{n}\right\}$ is defined as follows:

$$
\alpha_{n}=\lambda_{n} \frac{f\left(x_{n}\right)-f^{*}}{5 M^{2} L^{2}}
$$

with $0<\underline{\lambda} \leqslant \lambda_{n} \leqslant \bar{\lambda}<2$, which is inspired by Polyak in [16] for the incremental method.

Theorem 3.2. Let $\left\{x_{n}\right\}$ be generated by the Algorithm 1.1 with dynamic stepsize (3.1) and assume (2.1) is met. Then we have 
(i) if $\mathrm{H}$ is a finite dimensional Hilbert space, then $\left\{x_{n}\right\}$ converges to an optimal solution $x^{*}$ of the problem (1.1);

(ii) if $\mathrm{H}$ is a infinite dimensional Hilbert space, then $\left\{x_{n}\right\}$ converges weakly to an optimal solution $x^{*}$ of the problem (1.1).

Proof. Using Lemma 2.3 with $x=\tilde{x} \in S$, one can get that

$$
\begin{aligned}
\left\|x_{n+1}-\tilde{x}\right\|^{2} & \leqslant\left\|x_{n}-\tilde{x}\right\|^{2}-2 \alpha\left(f\left(x_{n}\right)-f(\tilde{x})\right)+5 \alpha^{2} M^{2} L^{2} \\
& =\left\|x_{n}-\tilde{x}\right\|^{2}-2 \lambda_{n} \frac{\left(f\left(x_{n}\right)-f^{*}\right)^{2}}{5 M^{2} L^{2}}+\lambda_{n}^{2} \frac{\left(f\left(x_{n}\right)-f^{*}\right)^{2}}{5 M^{2} L^{2}} \\
& =\left\|x_{n}-\tilde{x}\right\|^{2}-\lambda_{n}\left(2-\lambda_{n}\right) \frac{\left(f\left(x_{n}\right)-f^{*}\right)^{2}}{5 M^{2} L^{2}} \\
& \leqslant\left\|x_{n}-\tilde{x}\right\|^{2}-\underline{\lambda}(2-\bar{\lambda}) \frac{\left(f\left(x_{n}\right)-f^{*}\right)^{2}}{5 M^{2} L^{2}} .
\end{aligned}
$$

Hence, $\left\|x_{n}-\tilde{x}\right\|$ is a decreasing sequence, it follows that $\lim _{n \rightarrow \infty}\left\|x_{n}-\tilde{x}\right\|$ exists. Furthermore, taking the limit on both sides of the above inequality, one have $\lim _{n \rightarrow \infty} f\left(x_{n}\right)=f^{*}$ and then $\lim _{n \rightarrow \infty} \alpha_{n}=0$.

Next, we will prove that

$$
\lim _{n \rightarrow \infty}\left\|P_{C_{i}} x_{n}-x_{n}\right\|=0, i=1,2, \ldots, N .
$$

In fact, applying the convex of norm and the properties of the projections $P_{C_{i}}$, we have for each $\tilde{x} \in S$,

$$
\begin{aligned}
\left\|x_{n+1}-\tilde{x}\right\|^{2}=\left\|\sum_{i=1}^{N} \beta_{i} P_{C_{i}}\left(x_{n, M}\right)-\tilde{x}\right\|^{2} & \leqslant \sum_{i=1}^{N} \beta_{i}\left\|P_{C_{i}}\left(x_{n, M}\right)-\tilde{x}\right\|^{2} \\
& \leqslant \sum_{i=1}^{N} \beta_{i}\left(\left\|x_{n, M}-\tilde{x}\right\|^{2}-\left\|P_{C_{i}}\left(x_{n, M}\right)-x_{n, M}\right\|^{2}\right) \\
& =\left\|x_{n, M}-\tilde{x}\right\|^{2}-\sum_{i=1}^{N} \beta_{i}\left\|P_{C_{i}}\left(x_{n, M}\right)-x_{n, M}\right\|^{2}
\end{aligned}
$$

which implies that

$$
\sum_{i=1}^{N} \beta_{i}\left\|P_{C_{i}}\left(x_{n, M}\right)-x_{n, M}\right\|^{2} \leqslant\left\|x_{n, M}-\tilde{x}\right\|^{2}-\left\|x_{n+1}-\tilde{x}\right\|^{2} .
$$

Observing, for $1 \leqslant j \leqslant M$,

$$
\left\|x_{n, j}-x_{n}\right\|=\alpha_{n}\left\|h_{j}\right\|=\alpha_{n}\left\|\sum_{k=1}^{j} \omega_{k, j}(\mu) \nabla f_{k}\left(x_{n, j-1}\right)\right\| \leqslant \alpha_{n} M L \rightarrow 0(n \rightarrow \infty) .
$$

Moreover, by Lemma 2.3, $\lim _{n \rightarrow \infty}\left\|x_{n}-\tilde{x}\right\|$ exists for $\tilde{x} \in S$. Therefore, according to (3.2), we have

$$
\lim _{n \rightarrow \infty} \sum_{i=1}^{N} \beta_{i}\left\|P_{C_{i}}\left(x_{n, M}\right)-x_{n, M}\right\|^{2}=0 \text {. }
$$

Again using $\lim _{n \rightarrow \infty}\left\|x_{n, j}-x_{n}\right\|=0$, one can get

$$
\lim _{n \rightarrow \infty}\left\|P_{C_{i}} x_{n}-x_{n}\right\|=0, i=1,2, \ldots, N .
$$

At last, we will prove that $\left\{x_{n}\right\}$ converges weakly to an optimal solution $x^{*}$ of the problem (1.1).

In fact, if $\hat{x}$ is a weak-cluster point of the sequence $\left\{x_{n}\right\}$, using (3.3) and Lemma 2.1 we have $\hat{x} \in C=$ $\cap_{i=1}^{N} C_{i}$. Moreover, since $\lim _{n \rightarrow \infty} f\left(x_{n}\right)=f^{*}$, it follows that $\hat{x} \in S$. Hence, using Lemma 2.1, we have $\left\{x_{n}\right\}$ converges weakly to an optimal solution $x^{*}$ of the problem (1.1).

For the case of $\mathrm{H}$ being a finite dimensional Hilbert space, the result is clear. 


\subsection{Dynamic stepsize for unknown $\mathrm{f}^{*}$}

In the practical problems, the optimal value $f^{*}$ is usually unknown. In this subsection, we modify the dynamic stepsize (3.1) by replacing the optimal value $f^{*}$ with an estimate for the $n$th iteration as follows:

$$
\alpha_{\mathrm{n}}=\lambda_{\mathrm{n}} \frac{\mathrm{f}\left(\mathrm{x}_{\mathrm{n}}\right)-\mathrm{f}_{\mathrm{n}}^{*}}{5 \mathrm{M}^{2} \mathrm{~L}^{2}}, 0<\underline{\lambda} \leqslant \lambda_{\mathrm{n}} \leqslant \bar{\lambda}<2,
$$

where $f_{n}^{*}$ is the best value of $\min _{0 \leqslant k \leqslant n} f\left(x_{k}\right)$ achieved up to the $n$th iteration minus a positive number $\delta_{n}$ which is adjusted based on the iteration progress and is defined as follows:

$$
f_{n}^{*}=\min _{0 \leqslant k \leqslant n} f\left(x_{k}\right)-\delta_{n}
$$

and choose an initial value $\delta_{0}>0$ arbitrarily, let $\beta<1, \delta$ are fixed positive constant, then $\delta_{n}$ is updated by

$$
\delta_{n+1}= \begin{cases}\delta_{n}, & f\left(x_{n+1}\right) \leqslant f_{n}^{*}, \\ \max \left\{\beta \delta_{n}, \delta\right\}, & f\left(x_{n+1}\right)>f_{n}^{*} .\end{cases}
$$

Note that $\delta_{n}$ is adjusted based on the iteration progress, whenever $f\left(x_{n+1}\right) \leqslant f_{n}^{*}$ we increase $\delta_{n+1}$, or $\delta_{n+1}$ approaches to $\delta$. Furthermore, since $f_{n}^{*}=\min _{0 \leqslant k \leqslant n} f\left(x_{k}\right)-\delta_{n}$, it follows that $f\left(x_{n}\right)-f_{n}^{*} \geqslant \delta_{n} \geqslant \delta$. So $\alpha_{n}=\lambda_{n} \frac{f\left(x_{n}\right)-f_{n}^{*}}{5 M^{2} L^{2}} \geqslant \lambda_{5 M^{2} L^{2}}$, which is bounded away from zero. Hence, we have the following convergence property which is similar to Proposition 3.1.

Proposition 3.3. Let $\left\{x_{n}\right\}$ be generated by the Algorithm 1.1 with the dynamic stepsize $\left\{\alpha_{n}\right\}$ in (3.4). And assume the boundedness of gradient (i.e., (2.1) is met). Then we have

(i) if $f^{*}>-\infty$, then $\inf _{n \geqslant 0} f\left(x_{n}\right) \leqslant f^{*}+\delta$, where $L$ is given in (2.1) and $M$ is the number of component functions;

(ii) if $f^{*}=-\infty$, then $\inf _{n \geqslant 0} f\left(x_{n}\right)=-\infty$.

Proof. If it is not, we assume $\inf _{n \geqslant 0} f\left(x_{n}\right)>f^{*}+\delta$. Then there exists $\tilde{x} \in C$ such that

$$
\inf _{n \geqslant 0} f\left(x_{n}\right)-\delta>f(\tilde{x})
$$

Since $\delta_{n} \geqslant \delta$, without loss of generality, we can assume each time the target is attained (i.e., $f\left(x_{n+1}\right) \leqslant$ $\left.f_{n}^{*}\right)$. Then, the sequence $\min _{0 \leqslant k \leqslant n} f\left(x_{k}\right)$ decreases by at least $\delta$. And because of the $\inf _{n \geqslant 0} f\left(x_{n}\right)>f^{*}+\delta$, it easy to get that there exists $N$, such that $\delta_{n}=\delta$ for $n>N$.

By (3.5) and (3.6), we have

$$
f_{n}^{*}=\min _{0 \leqslant k \leqslant n} f\left(x_{k}\right)-\delta \geqslant \inf _{n \geqslant 0} f\left(x_{n}\right)-\delta>f(\tilde{x})
$$

for $n>N$. So

$$
f\left(x_{n}\right)-f(\tilde{x}) \geqslant f\left(x_{n}\right)-f_{n}^{*} .
$$

Using Lemma 2.3 with $x=\tilde{x}$, we have

$$
\begin{aligned}
\left\|x_{n+1}-\tilde{x}\right\|^{2} & \leqslant\left\|x_{n}-\tilde{x}\right\|^{2}-2 \alpha\left(f\left(x_{n}\right)-f(\tilde{x})\right)+5 \alpha^{2} M^{2} L^{2} \\
& \leqslant\left\|x_{n}-\tilde{x}\right\|^{2}-2 \lambda_{n} \frac{\left(f\left(x_{n}\right)-f_{n}^{*}\right)^{2}}{5 M^{2} L^{2}}+\lambda_{n}^{2} \frac{\left(f\left(x_{n}\right)-f_{n}^{*}\right)^{2}}{5 M^{2} L^{2}} \\
& =\left\|x_{n}-\tilde{x}\right\|^{2}-\lambda_{n}\left(2-\lambda_{n}\right) \frac{\left(f\left(x_{n}\right)-f_{n}^{*}\right)^{2}}{5 M^{2} L^{2}} \\
& \leqslant\left\|x_{n}-\tilde{x}\right\|^{2}-\underline{\lambda}(2-\bar{\lambda}) \frac{\left(f\left(x_{n}\right)-f_{n}^{*}\right)^{2}}{5 M^{2} L^{2}}
\end{aligned}
$$

Therefore, $\left\|x_{n+1}-\tilde{x}\right\|^{2} \leqslant\left\|x_{N}-\tilde{x}\right\|^{2}-(n+1-N) \underline{\lambda}(2-\bar{\lambda}) \frac{\left(f\left(x_{n}\right)-f_{n}^{*}\right)^{2}}{5 M^{2} L^{2}}$, when $n>N$, which is a contradiction when $n$ is large. Hence the assumption is false, and $\inf _{n \geqslant 0} f\left(x_{n}\right) \leqslant f^{*}+\delta$. 
At last we will modify the dynamic stepsize (3.1) by replacing the optimal value $f^{*}$ with another estimate $\mathrm{f}_{\mathrm{lev}}^{\mathrm{h}}$ for the $\mathrm{nth}$ iteration as follows:

$$
\alpha_{n}=\lambda_{n} \frac{f\left(x_{n}\right)-f_{\text {lev }}^{n}}{5 M^{2} L^{2}}, 0<\underline{\lambda} \leqslant \lambda_{n} \leqslant \bar{\lambda}<2,
$$

where $f_{\text {lev }}^{n}$ is constructed in the following algorithm, which is inspired by Goffin and Kiwiel in [9] for constrained convex minimization problem.

\section{Algorithm 3.4.}

Step 0: (Initiation). Select $x_{0} \in H, \delta_{0}>0, R>0$. Set $\sigma_{0}=0, f_{\text {lev }}^{(-1)}=\infty$. Set $n=0, l=0$, and $n_{0}=0\left(n_{l}\right.$ will denote the iteration number of the lth change of $f_{\text {lev }}^{\text {n }}$ ).

Step 1: (Objective evaluation). Calculate $f\left(x_{n}\right)$, set $f_{\text {rec }}^{n}=\min _{0 \leqslant k \leqslant n} f\left(x_{k}\right)$.

Step 2: (Sufficient descent detection). If $f\left(x_{n}\right) \leqslant f_{\text {rec }}^{n_{l}}-\frac{\delta_{l}}{2}$, set $n_{l+1}=n_{l}, \sigma_{n}=0, \delta_{l+1}=\delta_{l}$, increase $l$ by 1 , and go to Step 4.

Step 3: (Oscillation detection). If $\sigma_{\mathfrak{n}}>R$, set $n_{l+1}=n_{l}, \sigma_{\mathfrak{n}}=0, \delta_{l+1}=\frac{\delta_{l}}{2}$, increase $l$ by 1 .

Step 4: (Level update). Set $f_{\text {lev }}^{n}=f_{\text {rec }}^{n_{l}}-\delta_{l}$, and calculate the stepsize $\alpha_{n}$.

Step 5: (Path update). Set $\sigma_{n+1}=\sigma_{n}+C \alpha_{n}$, increase $n$ by 1 and go to step 1 .

Let $u$ split the iterations into groups $G_{l}=\left\{n_{l}, n_{l}+1, \ldots n_{l+1}-1\right\}, l \geqslant 0$. Within group $G_{l}$, the algorithm uses the same target level $f_{\text {lev }}^{n}=f_{\text {rec }}^{n_{l}}-\delta_{l}$ for $n \in G_{l}$. The level is updated only by sufficient descent (step 2) or oscillation (step 3) appeared.

The following proposition shows that the target levels $\mathrm{f}_{\mathrm{lev}}^{\mathrm{n}}$ are updated infinitely, (i.e., $l$ is increased infinitely), and $\inf _{n \geqslant 0} f\left(x_{n}\right)=\infty$ if $\delta_{l}$ is not diminishing.

Proposition 3.5. Let $\left\{x_{n}\right\}$ be generated by the Algorithm 1.1 with the dynamic stepsize $\left\{\alpha_{n}\right\}$ in (3.5). And assume the boundedness of gradient (i.e., (2.1) is met). Then $l \rightarrow \infty$, and $\lim _{l \rightarrow \infty} \delta_{l}=0$ or $\inf _{n \geqslant 0} f\left(x_{n}\right)=\infty$.

Proof. Firstly, we will prove $l \rightarrow \infty$ (i.e., $l$ is increased infinitely). In fact, if not, $l$ has only finite values, $l=0,1, \ldots \bar{l}$. According to step 4 and step 5 in the Algorithms 3.4, we have $\sigma_{n+1}=\sigma_{n}+C \alpha_{n}<R$ for all $n>n_{\bar{l}}$. It follows that $\lim _{n \rightarrow \infty} \alpha_{n}=0$. But according to step $4, \alpha_{n}=\lambda_{n} \frac{f\left(x_{n}\right)-f_{\text {lev }}^{n}}{5 M^{2} L^{2}} \geqslant \underline{\lambda_{i}} \frac{\delta_{\bar{l}}}{5 M^{2} L^{2}}>0$ when $\mathrm{n}>\mathrm{n}_{\bar{l}}$, which is a contradiction, so $\mathrm{l} \rightarrow \infty$.

Then, we prove $\lim _{l \rightarrow \infty} \delta_{l}=0$ or $\inf _{n \geqslant 0} f\left(x_{n}\right)=\infty$. In fact, if $\lim _{l \rightarrow \infty} \delta_{l}=\gamma>0$, from steps 2 and 3 we have there exists L such that $\delta_{l}=\gamma$ for $l>L$. And then according to step 2, we can get that $f_{\text {rec }}^{n_{l+1}}-f_{\text {rec }}^{n_{l}} \leqslant \frac{\gamma}{2}$. It follows that $\inf _{n \geqslant 0} f\left(x_{n}\right)=-\infty$.

At last, we will show that $\inf _{n \geqslant 0} f\left(x_{n}\right)=f^{*}$.

Theorem 3.6. Let $\left\{x_{n}\right\}$ be generated by the Algorithm 1.1 with the dynamic stepsize $\left\{\alpha_{n}\right\}$ in (3.5). And assume the boundedness of gradient (i.e., (2.1) is met) and $\inf _{n \geqslant 0} f\left(x_{n}\right)>-\infty$. Then $\inf _{n \geqslant 0} f\left(x_{n}\right) \leqslant f^{*}$.

Proof. If it is not, $\inf _{n \geqslant 0} f\left(x_{n}\right)>f^{*}$, and there exists $\tilde{x} \in C, \varepsilon_{0}>0$ such that

$$
\inf _{n \geqslant 0} f\left(x_{n}\right)>f(\tilde{x})+\varepsilon_{0} .
$$

According to Proposition 3.5, $\lim _{l \rightarrow \infty} \delta_{l}=0$. There exists L such that $\delta_{l}<\varepsilon_{0}$ for all $l>$ L. Hence,

$$
f_{\text {lev }}^{\mathrm{n}}=\mathrm{f}_{\text {rec }}^{\mathrm{n}_{l}}-\delta_{l} \geqslant \inf _{\mathrm{n} \geqslant 0} f\left(x_{n}\right)-\varepsilon_{0}>f(\tilde{x})
$$


for all $\mathrm{n}>\mathrm{n}_{\mathrm{L}}$. Moreover, using Lemma 2.3 with $x=\tilde{x}$, we have

$$
\begin{aligned}
\left\|x_{n+1}-\tilde{x}\right\|^{2} & \leqslant\left\|x_{n}-\tilde{x}\right\|^{2}-2 \alpha\left(f\left(x_{n}\right)-f(\tilde{x})\right)+5 \alpha^{2} M^{2} L^{2} \\
& \leqslant\left\|x_{n}-\tilde{x}\right\|^{2}-2 \lambda_{n} \frac{\left(f\left(x_{n}\right)-f_{\text {lev }}^{n}\right)^{2}}{5 M^{2} L^{2}}+\lambda_{n}^{2} \frac{\left(f\left(x_{n}\right)-f_{\text {lev }}^{n}\right)^{2}}{5 M^{2} L^{2}} \\
& =\left\|x_{n}-\tilde{x}\right\|^{2}-\lambda_{n}\left(2-\lambda_{n}\right) \frac{\left(f\left(x_{n}\right)-f_{\text {lev }}^{n}\right)^{2}}{5 M^{2} L^{2}} \\
& \leqslant\left\|x_{n}-\tilde{x}\right\|^{2}-\underline{\lambda}(2-\bar{\lambda}) \frac{\left(f\left(x_{n}\right)-f_{\text {lev }}^{n}\right)^{2}}{5 M^{2} L^{2}} .
\end{aligned}
$$

Hence,

$$
\frac{\lambda(2-\bar{\lambda})}{5 M^{2} L^{2}} \sum\left(f\left(x_{n}\right)-f_{l e v}^{n}\right)^{2}<\infty
$$

An then

$$
\sum_{n \geqslant 0} \alpha_{n}^{2}<\infty
$$

On the other hand, set $I=\left\{l, \delta_{l}=\frac{\delta_{l-1}}{2}\right\}$, since $\lim _{l \rightarrow \infty} \delta_{l}=0$, I is an infinite set. For $l+1 \in I, n_{l+1}=n$ in step 3, we have $\sigma_{n}=\sigma_{n-1}+C \alpha_{n-1}=\sum_{k=n_{l}}^{n-1} C \alpha_{k}>$ B. So for $l \in I$,

$$
\sum_{k=n_{l-1}}^{n_{l}-1} \alpha_{k}>\frac{B}{C} \text {. }
$$

Note that

$$
\sum_{n=1}^{\infty} \alpha_{n} \geqslant \sum_{l \in I} \sum_{k=n_{l-1}}^{n_{l}-1} \alpha_{k}
$$

and I is an infinite set, it follows that

$$
\sum_{n=1}^{\infty} \alpha_{n}=\infty .
$$

Using (3.7), (3.8), and the Proposition 3.3 in [19], we have $\lim _{\inf } f_{n \rightarrow \infty} f\left(x_{n}\right)=f^{*}$, which is contradiction with $\inf _{n \geqslant 0} f\left(x_{n}\right)>f^{*}$.

\section{4. conclusion}

In this paper, we research the convergence properties of Algorithm 1.1 for three types of stepsize rules: constant stepsize, dynamic stepsize for known $f^{*}$, and dynamic stepsize for unknown $f^{*}$. For case of dynamic stepsize for known $f^{*}$, we get that the Algorithm 1.1 converges weakly to an optimal solution $x^{*}$ of the problem (1.1).

\section{Acknowledgment}

This research is supported by NSFC Grants No:11301379; No:11226125; No:11671167.

\section{References}

[1] H. H. Bauschke, J. M. Borwein, On projection algorithms for solving convex feasibility problems, SIAM Rev., 38 (1996), 367-426.

[2] D. P. Bertsekas, Nonlinear Programming, Athena Scientific, Belmont, MA, (1995).

[3] D. P. Bertsekas, Incremental Gradient, Subgradient, and Proximal Methods for Convex Optimization: A Survey, MIT Press, Cambridge, (2011). 1, 1

[4] D. P. Bertsekas, Incremental aggregated proximal and augmented Lagrangian algorithms, arXiv preprint, 2015 (2015), 38 pages. 1 
[5] D. P. Bertsekas, J. N. Tsitsikls, Neuro-Dynamic Programming, Athena Scientific, Belmont, MA, (1996).

[6] P. L. Combettes, Hilbertian convex feasibility problem: convergence of projection methods, Appl. Math. Optim., 35 (1997), 311-330.

[7] K. Geobel, W. A. Kirk, Topics in Metric Fixed Point Theory, Cambridge University Press, Cambridge, (1990). 2.1

[8] K. Geobel, S. Reich, Uniform convexity, hyperbolic geometry, and nonexpansive mappings, Marcel Dekker, Inc., New York, (1984). 2.1

[9] J.-L. Goffin, K. C. Kiwiel, Convergence of a simple subgradient level method, Math. Program., 85 (1999), 207-211. 3.3

[10] T. Hastie, R. Tibshirani, J. Friedman, The elements of statistical learning. Data mining, inference, and prediction, Second edition, Springer, New York, (2009). 1

[11] K. C. Kiwiel, P. O. Lindberg, Parallel subgradient methods for convex optimization, Inherently parallel algorithms in feasibility and optimization and their applications (Haifa, 2000), 335-344, Stud. Comput. Math., North-Holland, Amsterdam, (2001). 1

[12] K. C. Krzysztof, Convergence of approximate and incremental subgradient methods for convex optimization, SIAM J. Optim., 14 (2003), 807-840. 1

[13] Z.-Q. Luo, On the convergence of the LMS algorithm with adaptive learning rate for linear feedforward networks, Neural Computation, 3 (1991), 226-245.

[14] A. Nedić, D. P. Bertsekas, Incremental subgradient methods for nondifferentiable optimization, SIAM J. Option, 12 (2001), 109-138. 1

[15] A. Nedić, D. P. Bertsekas, V. S. Borkar, Distributed Asynchronous Incremental Subgradient Methods, Stud. Comput. Math., 8 (2001), 381-407. 1, 1

[16] B. T. Polyak, Minimization of unsmooth functionals, USSR Comput. Math. \& Math. Phys., 9 (1969), 509-521. 3.2

[17] B. T. Polyak, Introduction to Optimization, Translated from the Russian. With a foreword by Dimitri P. Bertsekas. Translations Series in Mathematics and Engineering, Optimization Software, Inc., Publications Division, New York, (1987).

[18] F. Schöpfer, T. Schuster, A. K. Louis, An iterative regularization method for the solution of the split feasibility problem in Banach spaces, Inverse Problems, 2008 (2008), 20 pages. 2.2

[19] L. Y. Shi, Q. H. Ansari, C. F. Wen, J. C. Yao, A new algorithm for constrained composite minimization, J. Nonlinear Var. Anal., 1 (2017), 253-264. 1, 1.1, 2, 3.3

[20] S. Sra, S. Nowozin, S. J. Wright, Optimization for machine learning, MIT Press, Cambridge, Massachusetts, (2011). 1

[21] H.-K. Xu, Averaged mappings and the gradient-projection algorithm, J. Optim. Theory Appl., 150 (2011), 360-378. 1

[22] X. Yang, H.-K. Xu, Projection algorithms for composite minimization, Carpathian J. Math., 33 (2017), 389-397. 1, 1.1

[23] X. Zhao, P. B. Luh, J. Wang, Surrogate gradient algorithm for Lagrangian relaxation, J. Optim. Theory Appl., 100 (1999), 699-712. 1 